\title{
The effect of folic acid in women with recurrent pregnancy loss, polycystic ovary syndrome and hyperhomocysterinemia
}

\begin{abstract}
Introduction: Polycystic ovary syndrome (PCOS) is one of the major causes of infertility Worldwide and affects about $20 \%$ of infertile couples. Patients with PCOS have an increased risk of first trimester spontaneous abortion ranging from $25 \%$ to $73 \%$. Repeated pregnancy loss (RPL), specified as two or more consecutive pregnancy losses before the 20 th week of pregnancy is a frequent obstetric complication. In spite of this, $50 \%$ of cases the pathophysiology remains unknown
\end{abstract}

\begin{abstract}
Aim of the work: The purpose of this work was to prevent early recurrent pregnancy loss in adult females with polycystic ovary syndrome and hyperhomocysteinemia by using folic acid $5 \mathrm{mg} /$ daily.
\end{abstract}

Methods: The current study was carried out on 80 pregnant women in reproductive period, their age ranged from 20 to 35 ages. They were assorted into two groups: group one studying 40 women having a history of RPL with PCOS and group two including 40 adult females having a history of RPL and non PCOS. The women in the two groups were treated with $5 \mathrm{mg} /$ day folic acid/day for 20 weeks.

Results: Homocysteine level in patients with RPL and polycystic ovary was significantly higher than in women without polycystic ovary syndrome. In addition, folic acid administration at a daily dosage of $5 \mathrm{mg}$ reduced significantly the rate of fetal loss.
Volume 7 Issue 3 - 2018

\section{Allam IA, Dawood AS, El-Hawary TM, EL- Gharib MN \\ Department of Obstetrics \& Gynecology, Tanta University, Egypt}

Correspondence: Mohamed EL-Gharib, Department of Obstetrics \& Gynecology, Tanta University, Albahr Street, Egypt, Tel +201 I I7040040, Email mohelgharib@gmail.com

Received: June 06, 2018 | Published: June 29, 2018

Conclusion: We found that folic acid supplementation early in pregnancy, in a pharmacological dose might have a beneficial effect on pregnancy outcome.

\section{Introduction}

Hibbard in 1964 was the first to describe an association between spontaneous abortion and maternal folate deficiency due to defective folate metabolism. ${ }^{1}$ Homocysteine (Hcy) is an essential amino acid required for the growth of cells and tissues. Homocysteine (Hcy) is an intermediate product formed by the breakdown of methionine the aid of certain B-vitamins. Homocysteine remethylation to methionine is dependent on both folate and cobalamin; and may undergo trans-sulfuration to cysteine and cystathionine. ${ }^{2}$ Enzyme deficiencies involved in the remethylation of homocysteine result in hyperhomocysteinemia. ${ }^{3}$ Elevated homocysteine can occur in cases of dietary or genetic vitamin deficiency, or reduced enzyme activities. ${ }^{4}$ Homocysteine levels are typically higher in men than women, and increase with age. Common levels in Western populations are 10 to $12 \mu \mathrm{mol} / \mathrm{L}$, and levels of $20 \mu \mathrm{mol} / \mathrm{L}$ are found in populations with low B-vitamin intakes or in the elderly. ${ }^{5}$ Abnormally high levels of homocysteine in the serum, above $15 \mu \mathrm{mol} / \mathrm{L}$, are a medical condition called hyperhomocysteinemia. ${ }^{6}$ Elevated plasma homocysteine levels are associated with an increased risk of atherosclerosis and thrombosis, as well as a variety of other pathologies such as birth defects, osteoporosis, diabetes and renal disease. The prognostic ability of plasma homocysteine level as a hazard factor for atherothrombotic orders raised the appealing hypothesis that reduction of homocysteine levels by vitamin supplementation might result in a commensurate reduction and the risk of atherothrombotic events. ${ }^{7}$ The purpose of this study was to assess the prevention of recurrent pregnancy loss in females with polycystic ovary syndrome and hyperhomocysteinemia by administration of folic acid 5 milligram/day.

\section{Patients and methods}

This is a randomized blind comparative study conducted on 80 patients in the Department of Obstetrics \& Gynecology at Tanta University Hospital. All cases included in this study were selected early in pregnancy, immediately after pregnancy test was positive. The study began on May 2017 and end in May 2018. Patients were classified into two groups:

i. Group 1: Includes 40 patients with PCOS, hyperhomocysteinemia and a history of recurrent abortion, treated with $5 \mathrm{mg}$ /day folic acid/day for 20 weeks.

ii. Group 2: Includes 40 women with recurrent pregnancy loss without PCOS treated with folic acid $0.5 \mathrm{mg} /$ day.

The inclusion criteria were:

i. Age between 20 and 35 years old.

ii. Patients with PCO (PCOS was diagnosed according to the Rotterdam criteria if at least two of the following criteria were present: Oligo/amenorrhea, clinical or biochemical hyperandrogenism and PCO on USG)

iii. Patients with hyperhomocysteinemia.

iv. Patients with recurrent pregnancy loss (three or more consecutive miscarriages).

The exclusion criteria were:

a. Women with any systemic disease. 
b. Women have other causes of RPL, including chromosomal, uterine abnormalities; antiphospholipid syndrome, Inflammatory, autoimmune and any other condition affecting homeostasis, will be ruled out by either physical examination or patient history.

c. Smokers and alcohol consumption.

d. Patient with prolactinoma, congenital adrenal hyperplasia, Cushing syndrome, Virilizing ovarian or adrenal tumors.

All patients were subjected to the following:

i. An informed consent obtained from all participants in this research.

ii. Personal history: Name, age, parity, occupätion, residency and Special habits.

iii. Husband history: name, age, occupätion, residency, Special habits and chronic diseases.

iv. Past history: medical diseases, abdominal surgeries, drug therapy or allergy.

v. Menstrual history: menarche, regularity, duration, amount and associated päin.

vi. General \& local clinical examination: to exclude general diseases, local causes of RPL.

vii. Measurement of serum homocysteine by ELISA.

viii. Finally, using folic acid to evaluate its effect in prophylaxis against early recurrent pregnancy loss in women with polycystic ovary syndrome and hyperhomocysteinemia.

\section{Results}

The present work was carried out on 80 pregnant women in reproductive period, their age ranged from 20 to 35 years. They were assorted into two groups: Group one included 40 adult females having a story of repeated pregnancy loss (RPL) with PCOS. Group II included 40 adult females having a history of RPL and non PCOS. ). All participants completed the study and the primary outcome was analyzed. Both groups were well matched (Table 1) considering the age, gravidity, parity and BMI. However, homocysteine level in group 1 was significantly higher in the group:

i. Of patients with RPL and polycystic ovary than in group

ii. Of patients with RPL without polycystic ovary. In addition, the distribution of gravidity and parity was similarly insignificant (Table 2) \& (Table 3)

Table I The clinical data and homocyeine of the studied patients

\begin{tabular}{llll}
\hline Item & Group I & Group 2 & P \\
\hline Age & $28.88 \pm 4.85$ & $27.33 \pm 3.79$ & 0.866 \\
Gavidity & $4.73 \pm 0.64$ & $4.80 \pm 0.6 \mathrm{I}$ & 0.866 \\
Parity & $0.33 \pm 0.57$ & $0.53 \pm 0.64$ & 0.866 \\
BMI & $25.68 \pm 3.85$ & $26.23 \pm 3.9 \mathrm{I}$ & 0.866 \\
Homocysteine & $20.28 \pm 2.09$ & $8.99 \pm 1.76$ & $0.00 I^{*}$ \\
\hline
\end{tabular}

The effects of folic acid administration during the first 20 weeks of gestation on fetal outcome are depicted in Table 4. It was observed that administration of folic acid in homohycysteinemia-polycystic ovary cases caused significant decrease in the fetal loss.

Table 2 The distribution of gravidity in both groups

\begin{tabular}{|c|c|c|c|c|c|}
\hline \multicolumn{3}{|l|}{ Gravidity } & \multirow{2}{*}{$\begin{array}{l}\text { Group I } \\
15\end{array}$} & \multirow{2}{*}{$\begin{array}{l}\text { Group } 2 \\
12\end{array}$} & \multirow{2}{*}{$\begin{array}{l}\text { Total } \\
27\end{array}$} \\
\hline \multirow{2}{*}{4} & & $N$ & & & \\
\hline & & $\%$ & $37.50 \%$ & $30.00 \%$ & $33.80 \%$ \\
\hline \multirow{2}{*}{5} & & $\mathrm{~N}$ & 21 & 24 & 45 \\
\hline & & $\%$ & $52.50 \%$ & $60.00 \%$ & $56.30 \%$ \\
\hline \multirow{2}{*}{6} & & $N$ & 4 & 4 & 8 \\
\hline & & $\%$ & $10.00 \%$ & $10.00 \%$ & $10.00 \%$ \\
\hline \multirow{2}{*}{ Total } & & $\mathrm{N}$ & 40 & 40 & 80 \\
\hline & & $\%$ & $100.00 \%$ & $100.00 \%$ & $100.00 \%$ \\
\hline \multirow{2}{*}{ Chi-square } & $\times 2$ & \multicolumn{2}{|c|}{0.533} & & \\
\hline & P-value & \multicolumn{2}{|c|}{0.766} & & \\
\hline
\end{tabular}

Table 3 The distribution of parity in both groups

\begin{tabular}{|c|c|c|c|c|c|}
\hline Parity & & & Group I & Group 2 & Total \\
\hline \multirow{2}{*}{0} & & $N$ & 29 & 22 & 51 \\
\hline & & $\%$ & $72.50 \%$ & $55.00 \%$ & $63.80 \%$ \\
\hline \multirow{2}{*}{ I } & & $N$ & 9 & 15 & 24 \\
\hline & & $\%$ & $22.50 \%$ & $37.50 \%$ & $30.00 \%$ \\
\hline \multirow{2}{*}{2} & & $\mathrm{~N}$ & 2 & 3 & 5 \\
\hline & & $\%$ & $5.00 \%$ & $7.50 \%$ & $6.30 \%$ \\
\hline \multirow{2}{*}{ Total } & & $N$ & 40 & 40 & 80 \\
\hline & & $\%$ & $100.00 \%$ & $100.00 \%$ & $100.00 \%$ \\
\hline \multirow{2}{*}{ Chi-square } & $\times 2$ & \multicolumn{2}{|c|}{2.661} & & \\
\hline & P-value & \multicolumn{2}{|c|}{0.264} & & \\
\hline
\end{tabular}

Table 4 The effect of folic acid administration during the first 20 weeks of gestation on fetal outcome

\begin{tabular}{|c|c|c|c|c|c|c|c|}
\hline & & \multicolumn{2}{|c|}{$\begin{array}{l}\text { Group I } \\
(\mathrm{N}=40)\end{array}$} & \multicolumn{2}{|c|}{$\begin{array}{l}\text { Group } 2 \\
(\mathrm{~N}=40)\end{array}$} & \multirow[t]{2}{*}{$\mathbf{X}^{2}$} & \multirow[t]{2}{*}{ P-value } \\
\hline & & $\mathbf{N}$ & $\%$ & $\mathbf{N}$ & $\%$ & & \\
\hline \multirow{2}{*}{$\begin{array}{l}\text { Fetal } \\
\text { pulsations } \\
\text { by US GA } \\
(5-9)\end{array}$} & Positive & 38 & 95 & 32 & 80 & \multirow[b]{2}{*}{4.112} & \multirow[b]{2}{*}{$0.043^{*}$} \\
\hline & Negative & 2 & 5 & 8 & 20 & & \\
\hline \multirow{2}{*}{$\begin{array}{l}\text { Pregnancy } \\
\text { status GA } \\
(10-15)\end{array}$} & Positive & 35 & 92.1 & 23 & 71.9 & \multirow[b]{2}{*}{5.013} & \multirow[b]{2}{*}{$0.025 *$} \\
\hline & Negative & 3 & 7.9 & 9 & 28.1 & & \\
\hline \multirow{2}{*}{$\begin{array}{l}\text { Pregnancy } \\
\text { status GA } \\
(15-20)\end{array}$} & Positive & 34 & 97.1 & 18 & 78.3 & \multirow[b]{2}{*}{5.343} & \multirow[b]{2}{*}{$0.02 I^{*}$} \\
\hline & Negative & I & 2.9 & 5 & 23.17 & & \\
\hline \multirow{3}{*}{$\begin{array}{l}\text { Fetal } \\
\text { outcome }\end{array}$} & $\begin{array}{l}\text { Live } \\
\text { birth }\end{array}$ & 34 & 85 & 18 & 45 & \multirow{3}{*}{14.142} & \multirow{3}{*}{$0.001 *$} \\
\hline & Abortion & 5 & 12.5 & 17 & 42.5 & & \\
\hline & IUFD & I & 2.5 & 5 & 12.5 & & \\
\hline
\end{tabular}

$\mathrm{GA}=$ Gestational age in weeks 


\section{Discussion}

In the current work, we found that fasting serum homocyseine levels ranged between 15-25 and 5-12Umol $\backslash 1$ with a mean of $20.28 \pm 2.09$ and $8.99 \pm 1.76$ for group 1 and group 2 respectively. Homocysteine was significantly higher in women with RPL and polycystic ovary than in women without polycystic ovary but have RPL. Various surveys were focused on the connection between PCOS and insulin resistance. ${ }^{8}$ In addition, a high risk of premature coronary artery disease (CAD) has been described in patients with PCOS. ${ }^{9}$ However, the exact etiology has not even been fully identified. To identify the pathogenesis for CAD in PCOS patients, several genetic studies have related to risk factors for CAD. ${ }^{10,11}$ The risk factors associated with CAD are smoking, diabetes, family history, and increased homocysteine (Hcy) levels in PCOS. ${ }^{12}$ Among the risk factors, it was described that the homocysteine level is extremely linked to insulin resistance in PCOS patients. ${ }^{13}$ In Kazerooni and associates study, they proved to investigate the role of hyperandrogenemia, insulin resistance, and hyperhomocysteinemia as well as inherited and acquired thrombophilias in patients with PCOS who had recurrent pregnancy loss (RPL). The writers establish that patients with PCOS and RPL had higher testosterone, DHEAS, and homocysteine (Hcy) levels than patients without PCOS but with RPL. RPL in those patients with PCOS was also associated with hypofibrinolysis and decreased insulin sensitivity. Kazerooni and coworkers, also observed higher levels of Hcy in patients with RPL than in healthy people. ${ }^{14}$

In the present study folic acid was given in an oral daily dose of $5 \mathrm{mg} /$ day to women of group 1 and in a dose of $0.5 \mathrm{mg} /$ day for women of group 2. We found that the effect of folic acid $5 \mathrm{mg} /$ day was significant while the effect of folic acid $0.5 \mathrm{mg}$ was non-significant against the occurrence of early recurrent pregnancy loss $(p<0.05)$. The homocysteine levels of hyperhomocysteinemic women with PCOS were reduced after 3 months of folic acid supplementation, and the rate of decrease was higher among women without insulin resistance. ${ }^{15}$ Randeva and associates noted that elevated serum Hcy levels are present in PCOS women, indicating that an alteration in Hcy metabolism may be implicated in the increased risk of cardiovascular disease in such patients. ${ }^{16}$ In spite of several works, the real mechanism in which Hcy is increased in PCOS patients is not well-read. A bunch of factors affect serum Hcy levels, including age, gender, sexsteroid hormones, insulin resistance (IR), body mass index (BMI), and glucose tolerance and chronic inflammation. Increased insulin levels have been taken as a regulating factor of Hcy in which insulin inhibits hepatic cystathionine b-synthase activity that increases serum homocysteine. ${ }^{13}$ Follicular fluid also contains Hcy. Disorders of the composition of follicular fluid such as the microenvironment of an oocyte may influence its development. A primary deficiency of natural folate resulting in an increment of the total Hcy concentration may be detrimental to the character of the oocyte, subsequent fertilization, embryo quality, implantation, embryogenesis, and fetal outcome. ${ }^{17}$

Despite elevated serum insulin, insulin resistance, and homocysteine levels, and their effects on oocyte numbers and maturation in PCOS patients, there were no differences in follicular parameters and clinical pregnancy rates between hyperinsulinemic and hyperhomocysteinemic PCOS patients and the other groups..$^{18}$ In spite of several studies, the real mechanism in which Hcy is increased in PCOS patients is not well-understood. Increased insulin levels have been considered as a modulating factor of Hcy in which insulin inhibits hepatic cystathionine b-synthase activity that increases serum homocysteine. ${ }^{13}$ In addition, there was also a positive significant correlation between elevated serum insulin and IR in these patients. ${ }^{19}$ Several evidences reported higher levels of sex steroid hormones and androgens in PCOS patients, which may influence the metabolism of Hcy and increase its plasma levels. ${ }^{20}$ Lastly, we conclude that elevated homocysteine in PCOS patients is a hazard factor for recurrent pregnancy loss. Folic acid supplementation in early pregnancy, in a pharmacological dose, might have a beneficial effect on pregnancy outcome.

\section{Disclosure}

We announce that we did not get any funds from whatever individual or establishment. If the patient refused to finish the field, she was taken out and replaced by another one from who are satisfying the inclusion criteria of the survey. We did not classify the patients according to their religious belief or civilization or race or any other unrelated points. The authors declare no conflicts of interest in this study.

\section{Acknowledgements}

None.

\section{Conflict of interest}

Author declares that there is no conflict of interest.

\section{References}

1. Hibbard BM. The role of folic acid in pregnancy with particular reference to anemia, abruption and abortion. J Obstetrics and Gynaecol Br Commonw.1964;71:529-42.

2. Welch GN, Loscalzo J. Homocysteine and atherotrombosis. $N$ Engl $J$ Med. 1998;338(15):1042-50.

3. Burke G, Robinson $\mathrm{K}$, Refsum $\mathrm{H}$, et al. Intrauterine growth retardation, perinatal death and maternal homocysteine levels. $N$ Engl $J$ Med. 1992;326(1):69-70.

4. Nelen W, Blom HJ, Steegers EA, et al. Homocysteine and Folate Levels as Risk Factors for Recurrent Early Pregnancy Los. Obstet Gynecol. 2000;95(4):519-524

5. McLean RR, Jacques PF, Selhub J, et al. Homocysteine as a predictive factor for hip fracture in older persons. NEnglJMed. 2004;350(20):20429

6. Yang $\mathrm{G}, \mathrm{Wu} \mathrm{L}$, Jiang $\mathrm{B}$, et al. $\mathrm{H}_{2} \mathrm{~S}$ as a physiologic vasorelaxant: hypertension in mice with deletion of cystathionine $\gamma$-lyase. Science. 2008;322(5901):587-90.

7. Lentz SR. Mechanisms of thrombosis in hyperhomocysteinemia. Curr Opin Hematol. 1998;5(5):343-9.

8. Lee EJ, Oh B, Lee JY, et al. A single nucleotide polymorphism of INSR gene for polycystic ovary syndrome. Fertil Steril. 2008;89(5):1213-20.

9. Talbott EO, Zborowskii JV, Boudraux MY. Do women with polycystic ovary syndrome have an increased risk of cardiovascular disease? Review of the evidence. Minerva Ginecol. 2004;56(1):27-39.

10. Palep-Singh M, Picton HM, Yates ZR, et al. Plasma homocysteine concentrations and the single nucleotide polymorphisms in the methionine synthase gene (MTR 2756A > G): associations with the polycystic ovary syndrome. An observational study. Eur J Obstet Gynecol Reprod Biol. 2008;138(2):180-6. 
11. Oktem M, Ozcimen EE, Uckuyu A. Polycystic ovary syndrome is associated with elevated plasma soluble CD40 ligand, a marker of coronary artery disease. Fertil Steril. 2009;91(6):2545-2550.

12. Taylor BV, Oudit GY, Evans M. Homocysteine, vitamins, and coronary artery disease. Comprehensive review of the literature. Can Fam Physician. 2000;46:2236-45.

13. Badawy A, State O, El Gawad SSh, et al. Plasma homocysteine and polycystic ovary syndrome: the missed link. Eur J Obstet Gynecol Reprod Biol. 2007;131(1):68-72.

14. Kazerooni T, Ghaffarpasand F, Asadi N, et al. Correlation between thrombophilia and recurrent pregnancy loss in patients with polycystic ovary syndrome: A comparative study. J Chin Med Assoc. 2013;76(5):282-288.

15. Kazerooni T, Asadi N, Dehbashi S. Effect of folic acid in women with and without insulin resistance who have hyperhomocysteinemic polycystic ovary syndrome. International Journal of Gynecology and Obstetrics. 2008;101(2):156-160.

16. Randeva HS, Lewandowski KC, Drzewoski J, et al. Exercise decrease plasma total homocysteine in overweight young women with polycystic ovary syndrome. J Clin Endocrinol Metab. 2002;87(10):4496-501.
17. Ebisch IM, Thoma CM, Peters WH, et al. The importance of folate, zinc and antioxidants in the pathogenesis and prevention of subfertility. Hum Reprod Update. 2007;13(2):163-74.

18. Nafiye Y, Sevtap K, Muammer D, et al. The effect of serum and intrafollicular insulin resistance parameters and homocysteine levels of nonobese, nonhyperandrogenemic polycystic ovarysyndrome patients on in vitro fertilization outcome. Fertility and Sterility. 2010;93(6): 1864-1869.

19. Esmaeilzadeh S, Tahmasbpour E, Gholinezhad-Chari M. Hyperhomocysteinemia, insulin resistance and body mass index in Iranian young women with polycystic ovary syndrome. Middle East Fertility Society. 2017;22(2):149-155.

20. Sachan R, Patel M, Gupta P, et al. Correlation of plasma homocysteine levels with BMI and insulin resistance, amongst obese, overweighted and non obese infertile women. International Journal of Scientific and Research Publications. 2012;2(5):1-6. 